\title{
RHIC Spin Flipper Commissioning
}

\author{
M. Bai*, A.U. Luccio*, W.W. MacKay*, V. Ranjbar ${ }^{\dagger}$ and T. Roser* \\ ${ }^{*} B N L$, Upton, NY 11973, USA \\ ${ }^{\dagger}$ Indiana University, Bloomington, IN 47408, USA
}

\begin{abstract}
An ac dipole with horizontally oriented oscillating magnetic field (spin flipper) was installed in RHIC to reverse the spin direction in the presence of two full Siberian snakes, thereby reducing the systematic errors for the spin physics experiments in RHIC. With two full snakes, the spin vector completes one full precession around the vertical direction in two revolutions, and the spin depolarization resonances due to the machine imperfections and betatron oscillations are eliminated. Since the spin flipper provides an oscillating horizontal dipole field, a "spin resonance" can occur if the spin flipper frequency is placed in the neighborhood of the spin precession frequency $[1,2,3]$. By slowly sweeping the spin flipper frequency across the spin precession frequency, a full spin flip can be achieved. This paper reports the results of the RHIC spin flipper commissioned during the RHIC 2002 polarized proton run. By running the spin flipper at a slightly different configuration, one can also measure the spin precession tune.
\end{abstract}

\section{INTRODUCTION}

Like any other magnets, the spin motion through an ac dipole also obeys the ThomasBMT equation

$$
\frac{d \vec{S}}{d t}=\frac{e}{\gamma m}(1+G \gamma) \vec{S} \times \vec{B}(t),
$$

where $\vec{S}$ is the spin vector in the particle rest frame, $G$ is the anomalous gyromagnetic g-factor and $\gamma m c^{2}$ is the moving particle energy. $\vec{B}(t)$ is the magnetic field of the ac dipole

$$
B(t)=B_{o} \cos \left(2 \pi f_{m} t+\chi\right)
$$

where $B_{o}$ is the amplitude of the oscillating magnetic field, $f_{m}$ is the oscillation frequency and $\chi$ is the arbitrary phase of the ac dipole oscillating magnetic field.

In a perfect planar circular accelerator, the beam's spin vector precesses around vertical direction. With the ac dipole in the machine, the spin vector gets kicked away from the vertical direction every time it passes through the ac dipole. In the frame which rotates at the same oscillation frequency of $f_{m}$, the two-component spinor equation then becomes

$$
\frac{d \psi_{K}}{d \theta}=-\frac{i}{2}(\vec{\sigma} \cdot \vec{n}) \psi_{K}
$$

where

$$
\vec{n}=G \gamma \hat{e_{3}}-B_{o} \hat{e_{1}} \text {. }
$$

Here, $\hat{e_{1}}$ is the unit vector pointing radially outward, and the unit vector $\hat{e_{3}}$ points up. Compare this with the spinor equation of an intrinsic spin resonance [4]; the effect of

tWork performed under the auspices of US Department of Energy CP675, Spin 2002: ISth Int'l. Spin Physics Symposium and Workshop on Polarized Electron Sources and Polarimeters, edited by Y. I. Makdisi, A. U. Luccio, and W. W. MacKay (C) 2003 American Institute of Physics 0-7354-0136-5/03/\$20.00 
the ac dipole on the spin motion is equivalent to a spin resonance located at

$$
v_{s} \doteq v_{m}=\frac{f_{m}}{f_{\text {rev }}}
$$

where $v_{s}$ is the spin tune, $v_{m}$ is the ac dipole oscillation tune and $f_{r e v}$ is the beam revolution frequency. The strength of this ac-dipole-induced spin resonance is given by

$$
\varepsilon_{K}=\frac{1+G \gamma}{4 \pi} \frac{B_{o} L}{B \rho}
$$

where $L$ is the length of the ac dipole and $B \rho$ is the magnetic rigidity of the beam.

According to the Froissart-Stora formula, the beam polarization, after crossing through a spin resonance, is

$$
P_{f}=\left(2 e^{-\pi\left|\varepsilon_{K}\right|^{2} / 2 \alpha}-1\right) P_{0}
$$

where $P_{f}$ and $P_{0}$ are the beam polarization after and before the resonance. $\alpha=\frac{d \Delta v_{m}}{d \theta}$ is the resonance crossing rate, where $\Delta v_{m}$ is the width of the ac dipole oscillation tune sweep. To achieve a $99.999 \%$ spin flip, the following condition has to be fulfilled

$$
\alpha \leq 0.13\left|\varepsilon_{K}\right|^{2} .
$$

\section{SPIN FLIPPING IN RHIC}

\section{RHIC spin setup}

To eliminate the spin depolarization resonances along the acceleration, two pairs of Siberian snakes were installed in RHIC [5]. In each ring, the two snakes are placed on opposite sides of ring. Each snake consists of four super-conducting helical dipole magnets and rotates the spin vector by $180^{\circ}$ around an axis which lies in the horizontal plane and is called the snake axis. The spin precession tune is given by

$$
v_{s}=\frac{1}{\pi}\left|\psi_{1}-\psi_{2}\right|
$$

where $\psi_{i}$ is the angle between the axis of the $i_{t h}$ snake and the longitudinal direction. In general, the axes of the two snakes are configured to be $\pm 45^{\circ}$ away from the longitudinal direction for the RHIC polarized proton run and the nominal spin precession tune is $\frac{1}{2}$. In order to rotate the spin vector by $180^{\circ}$, the two outer helical magnets are powered with the same current with opposite polarities. Similarly the two inner helical magnets are powered with equal but opposite currents.

The RHIC beam polarization was measured with the CNI (Compton Nuclear Interference) polarimeter $[7,8,9]$ located at the IP12 region [6]. It measures the left-right asymmetry of the recoiling carbon nucleus. A typical measurement requires 2 million events to reach a $2 \%$ statistical error. 


\section{RHIC Spin Flip}

To induce a full spin flip in RHIC, one can sweep the artificial spin resonance across the spin precession frequency. In order to cross the resonance, one needs to detune the snake axis to move the spin precession tune away from $\frac{1}{2}$.

The RHIC spin flipper is located at the interaction region of IP4 and is common to both beams. Since each Siberian snake in RHIC is energized with two independent power supplies, the control of the spin precession tune in the two rings is independent of each other. Thus, one should in principle be able to achieve spin flipping in one ring without impacting the beam polarization in the other ring.

Two spin flips were tried in the Blue ring at the end of a typical polarized proton store. We first ramped both snakes in the Blue ring to the settings which correspond to $v_{s}=0.48$. Table 1 lists the snake inner and outer currents set values and read-backs. We

TABLE 1. Blue Siberian Snake Current Setting

\begin{tabular}{ccccc}
\hline & \multicolumn{2}{c}{$v_{s}=0.48$} & \multicolumn{2}{c}{$v_{s}=0.5$} \\
\hline magnet & set point[A] & readback[A] & set point[A] & readback[A] \\
\hline b03 snake outer & 106.11 & 105.75 & 99.95 & 99.72 \\
b03 snake inner & 325.06 & 324.07 & 325.06 & 324.33 \\
bi9 snake outer & 106.11 & 106.40 & 99.95 & 100.26 \\
bi9 snake inner & 325.06 & 324.88 & 325.94 & 326.09 \\
\hline
\end{tabular}

then ramped the ac dipole magnetic field amplitude from zero to 100 gauss-m in 6000 revolution turns with the resonant frequency fixed at $0.47 f_{\text {rev }}$. Here, $f_{\text {rev }}$ is the particle's revolution frequency around the ring. The ac dipole frequency was then swept from $0.47 f_{\text {rev }}$ to $0.49 f_{\text {rev }}$ in 200,000 revolution turns. The ac dipole was gradually turned off at a fixed frequency of $0.49 f_{\text {rev }}$ during 6000 turns. With the ac dipole maximum field of 100 gauss-m and the resonance crossing rate

$$
\alpha=\frac{d \Delta v_{m}}{d \theta}=\frac{0.02}{200000 \times 2 \pi}=1.6 \times 10^{-8},
$$

the expected spin flip is

$$
\frac{P_{f}}{P_{0}}=-1.0 \text {. }
$$

Fig. 1 shows the measured asymmetry before and after the spin flipping. The average spin flipping efficiency $\eta$ is defined as

$$
P_{i}=P_{0} \eta^{i}
$$

where $P_{0}$ is the beam polarization before the spin flipping and $P_{i}$ is the beam polarization after the $i^{\text {th }}$ spin flipping. After two spin flips, we measured an efficiency of

$$
\eta=0.66 \text {. }
$$

The fact that we did not reach full spin flip can be either due to the spin tune not being 0.48 as we expected or due to the spread of spin tune among the different particles in 


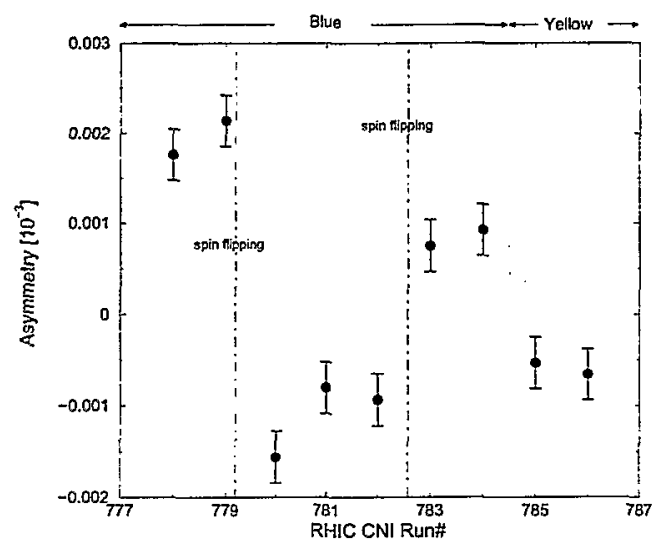

FIGURE 1. This plot shows the measured beam asymmetry before and after the spin fiipping in RHIC. The vertical averaged asymmetry in Blue was measured as $0.00195 \pm 0.0002$ After the 1st spin flipping, the asymmetry was measured as $0.0011 \pm 0.000164$. The asymmetry was $0.00084 \pm 0.0002$ after the $2 \mathrm{nd}$ spin flipping. The data point of RHIC CNI run 785 and 786 correspond to the measured asymmetry in the Yellow ring after the 2nd spin flipping in Blue.

the bunch. Particles with different betatron oscillation amplitudes experience different focusing forces; hence they have different spin precession frequencies. In general, the spin tune is a function of the betatron oscillation amplitude. Fig. 2 shows the numerical simulation of spin flipping of a beam with a normalized $95 \%$ emittance of $25 \pi \mathrm{mm}$-mrad emittance in both planes at $G \gamma=191.5$.

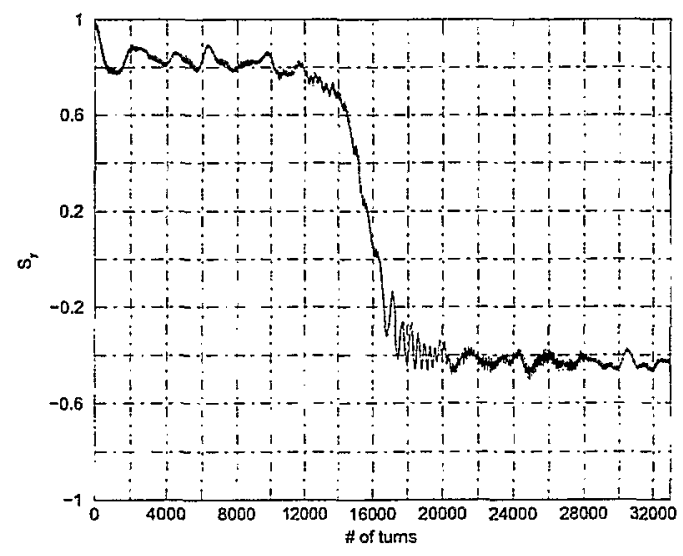

FIGURE 2. This plot shows the spin tracking results of spin flipping of 100 particles at $G \gamma=191.5$. They are gaussian distributed with $25 \pi \mathrm{mm}$-mrad in both horizontal and vertical planes. The final polarization after the flipping Only $40 \%$ of the initial polarization. With the same machine parameters and spin flipper setup, the single particle tracking yields a full spin flip.

The asymmetry in Yellow was also measured as before the spin flippings in the Blue as $0.0015 \pm 0.0003$ However, the fact that we measured the Yellow asymmetry as 
$-0.00075 \pm 0.0002$ after the 2nd spin flipping in the Blue as shown in Fig. 1 indicated that the spin tune in the Yellow ring was not. $\frac{1}{2}$. Otherwise, the spin flipping in the Blue ring should not have done any harm to the beam polarization even though the ac dipole affects both beams. This is also consistent with the two spin flipping attempts in the Yellow ring in which no spin flipping except depolarization was observed even though the same procedure as for the Blue ring was followed.

\section{CONCLUSION}

A short study of using the RHIC vertical ac dipole as a spin flipper was performed at the end of the RHIC 2002 polarized proton run. A partial spin flip was obtained in the RHIC Blue ring at the storage energy. However, due to the time limitation, we did not get chance to investigate the cause of depolarization and improve the spin flip efficiency. No spin flipping was obtained in the Yellow ring.

\section{ACKNOWLEDGMENTS}

The authors would like to thank Dr. G. Bunce, Dr. Y. Makdisi, Dr. L. Ahrens, Dr. H. Huang and Dr. N. Sato for the fruitful discussions and support. The authors would also like to thank A. Zaltsman, P. Oddo, C. Pai, T. Russo, R. Sanders, L. Hoff, J. Piacentino and B. Oerter for their technical support. This work is performed under the auspices of Department of Energy of U.S.

\section{REFERENCES}

1. D.D.Caussyn et al., 'Spin Flipping a Stored Polarized Proton Beam', Phys. Rev. Lett. 73, 2857 (1994).

2. B.B.Blinov et al., 'Spin Flipping in the Presence of a Full Siberian Snake', Phy. Rev. Lett. 81, 2906 (1998).

3. T. Roser, Handbook of Accelerator Physics and Engineering, P. 150, edited by A. Chao and M. Tigner.

4. S. Y. Lee, Spin Dynamics and Snakes in Synchrotrons, World Scientific, 1997

5. T. Roser et al. 'Accelerating and Colliding Polarized Protons in RHIC with Siberian Snakes', EPAC2002, June. 2002.

6. H. Hunag et al. 'Commissioning CNI Proton Polarimeters in RHIC', EPAC2002, June 2002.

7. O. Jinnouchi et al. 'RHIC pC CNI Polarimeter: Status and Performance from the First Collider Run', Proceedings of 15th International Spin Physics Symposium, BNL, 2002.

8. K. Kurita et al. 'RHIC pC CNI Polarimeter: Experimental Setup and Physics Results', Proceedings of 15th International Spin Physics Symposium, BNL, 2002.

9. G. Igo et al. 'Absolute Calibration of the CNI Polarimeters at RHIC Using $125 \mathrm{GeV} / \mathrm{A} \mathrm{C}$ Ions',Proceedings of 15th International Spin Physics Symposium, BNL, 2002. 
